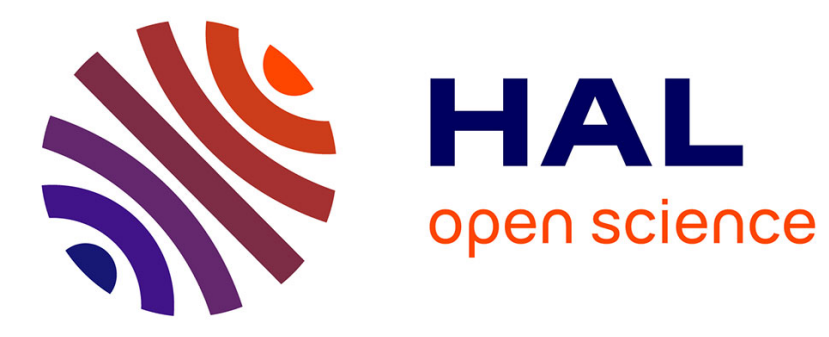

\title{
Optical parametric oscillation in 1D semiconductor microcavities
}

Vincenzo Ardizzone, Marco Abbarchi, Thimotée Lecomte, Aristide Lemaitre, Isabelle Sagnes, Pascale Senellart, Jacqueline Bloch, Philippe Roussignol, Jérôme Tignon

\section{To cite this version:}

Vincenzo Ardizzone, Marco Abbarchi, Thimotée Lecomte, Aristide Lemaitre, Isabelle Sagnes, et al.. Optical parametric oscillation in 1D semiconductor microcavities. physica status solidi (b), 2012, 249 (5), pp.896-899. 10.1002/pssb.201100115 . hal-00702857

\section{HAL Id: hal-00702857 https://hal.science/hal-00702857}

Submitted on 31 May 2012

HAL is a multi-disciplinary open access archive for the deposit and dissemination of scientific research documents, whether they are published or not. The documents may come from teaching and research institutions in France or abroad, or from public or private research centers.
L'archive ouverte pluridisciplinaire HAL, est destinée au dépôt et à la diffusion de documents scientifiques de niveau recherche, publiés ou non, émanant des établissements d'enseignement et de recherche français ou étrangers, des laboratoires publics ou privés. 


\title{
Optical parametric oscillaton in 1D semiconductor microcavities
}

\author{
Vincenzo Ardizzone ${ }^{*}, 1$, Marco Abbarchi ${ }^{1}$, Timothee Lecomte ${ }^{1}$, Aristide Lemaitre ${ }^{2}$, Isabelle Sagnes ${ }^{2}$, \\ Pascale Senellart $^{2}$, Jacqueline Bloch ${ }^{2}$, Philippe Roussignol ${ }^{1}$, Jerome Tignon ${ }^{1}$ \\ ${ }^{1}$ Laboratoire Pierre Aigrain, École Normale Supérieure, CNRS (UMR 8551), Université P. et M. Curie, Université D. Diderot, 75231 \\ Paris Cedex 05, France. \\ ${ }^{2}$ LPN/CNRS, Route de Nozay, F-91460 Marcoussis, France.
}

Received XXXX, revised XXXX, accepted XXXX

Published online XXXX

Key words: semiconductor micorcavity, optical parametric oscillation

${ }^{*}$ Corresponding author: e-mail vincenzo.ardizzone@lpa.ens.fr

We present an experimental investigation of parametric scattering processes and optical parametric oscillations in wire shaped, one-dimensional semiconductor microcavities. Far field emission patterns and corresponding band dispersion are studied by polarization resolved measurements and power dependence measurements under resonant and non-resonant excitation.
The multiplicity of the photonic bands allows for an efficient engineering of interbranch parametric scattering processes. We demonstrate the onset of optical parametric oscillation of perfectly balanced twin beams, degenerate in energy and split in momentum space.

Copyright line will be provided by the publisher

1 Introduction Recent advances in the fabrication and processing of high quality semiconductor microcavities demonstrated interesting and promising features of this peculiar kind of nanoemitters. Strong light-matter coupling and polariton condensation are two typical examples of phenomena well established in two dimensional GaAs microcavities (2D-MCs). A different application of semiconductor microcavities is the generation of parametric scattering processes (PSPs) and optical parametric oscillation (OPO).[1-6] From this point of view these systems are promising candidates for the realization of semiconductor micrometric sources of quantum-correlated photon beams. This kind of source is extremely important for applications in Quantum Information, like key distribution in Quantum Cryptography. [7,8]

First demonstrations of parametric phenomena in 2DMCs were based on the strong light-matter coupling [1, $9,10]$ where exciton (X) and cavity photon (Cav) can behave as two strongly coupled oscillators. The two new normal modes of the system are half-light, half-matter quasiparticles called upper polariton and lower polariton (UP and LP, respectively). Typical band dispersions in energy- angle space are shown in Fig. 1 a). The efficient realization of PSPs with energy and momentum conservation (phase matching conditions, PMCs) is based on the peculiar shape of the LP: when exciting at the inflection point of the Sshaped LP branch, S and I parametric oscillation can take place as schematically reported on top of Fig. 1a). [9,11] It is now well established that low-threshold OPO between non degenerate $\mathrm{S}$ and I beams in 2D-MCs can be achieved using very large $\chi^{3}$ polaritonic non-linearities. Nevertheless, this approach suffers from intrinsic drawbacks: $(i)$ as schematically shown in Fig. 1 a) and b) the excitation laser must be adjusted to a specific angle, (ii) the strong coupling (SC) can be achieved also at room temperature but there are still no evidences of parametric interactions at this temperature in strong-coupled samples and (iii) S and I beams are intrinsically strongly unbalanced, due to the great excitonic component of the idler. Moreover this difference in intensity between $\mathrm{S}$ and I makes difficult the study of quantum correlations between them.

A more suitable method for the realization of OPO in 2D-MCs has been demonstrated in triply resonant vertical MCs, based on interbranch parametric scattering. In this 
a)

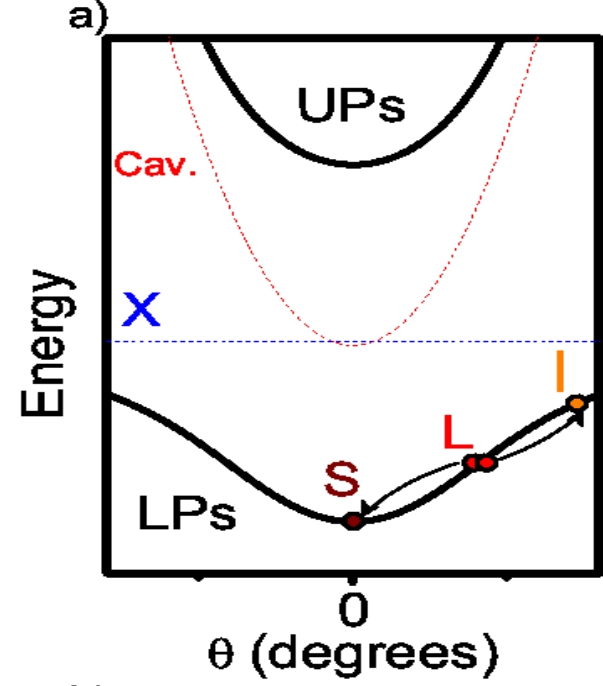

b)

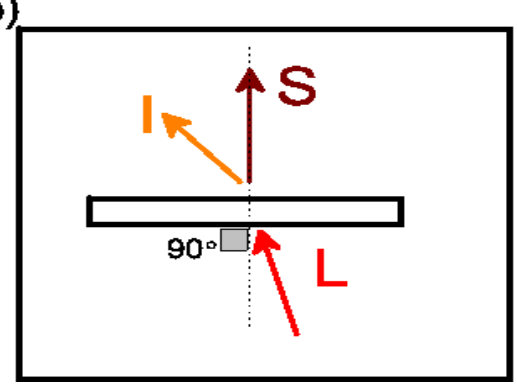

c)

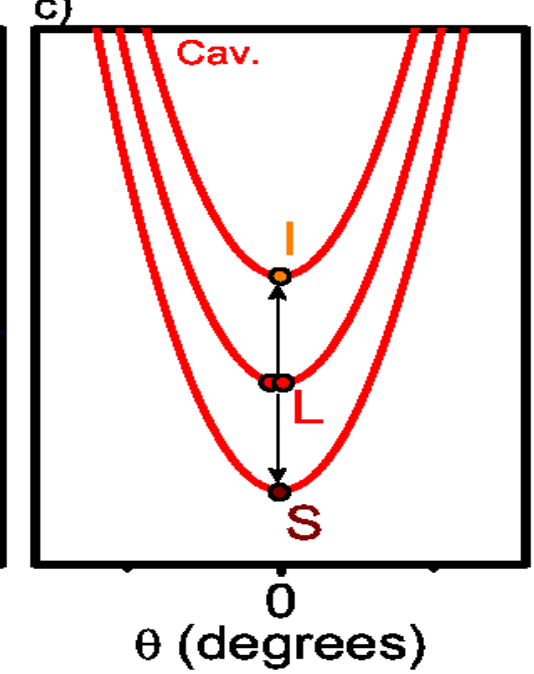

d)

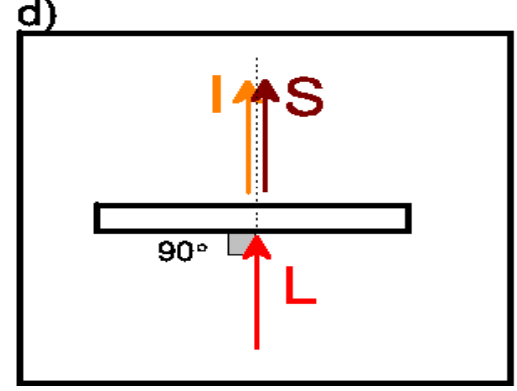

e)

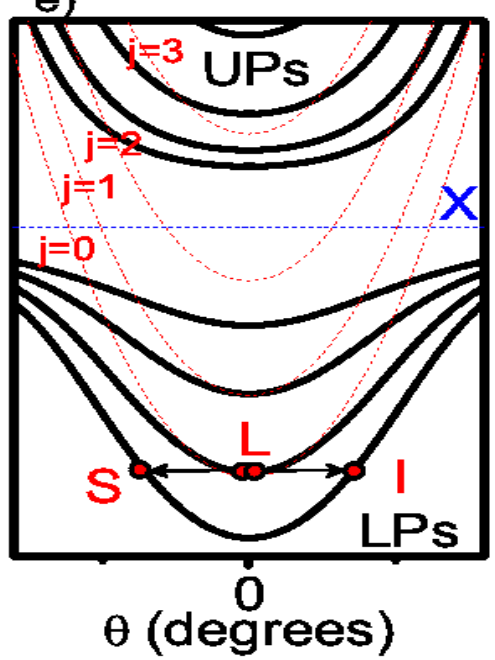

f)

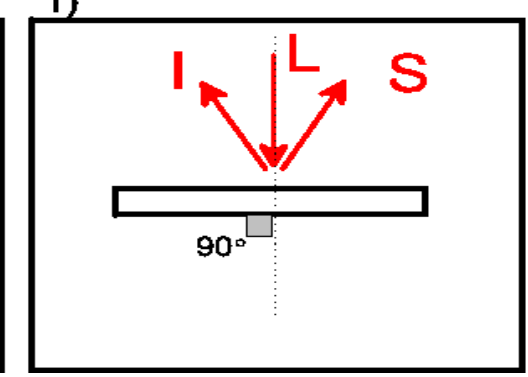

Figure $1 \mathrm{a}$ ) Thin lines are exciton (X) and cavity photon (Cav) dispersions. Thick lines are UP an LP dispersions. Arrows indicate the intrabranch parametric process. b) Scheme of the PSP: the excitation laser is tilted with respect to the normal to the sample surface while signal S and idler I are split. c) Photonic band dispersion of a triply-resonant 2D-MC and corresponding interbranch PSP split in energy and degenerate in momentum space. d) Corresponding scheme of the PSP with laser normal to the sample surface and degenerate $\mathrm{S}$ and I e) Thin lines are photonic band dispersion and exciton states. Thick lines are multi-branch UP and LP states. Arrows show a interbranch PSP degenerate in energy and split in momentum. f) Scheme of the PSP with laser normal to the sample surface and mirror-symmetric S and I.

situation, which is schematically shown in Fig. $1 \mathrm{c}$ ), the three coupled cavities have multiple photonic bands.[12] By tuning the relative energy of the three bands it is possible to generate twin $\mathrm{S}$ and I beams which are degenerate in momentum and split in energy (vertical process). This can be made by pumping at normal incidence at the energy of the intermediate cavity. This method allows relevant improvements in the realization of 2D-MCs-based OPO:[12, 13] strong coupling is no more necessary and multi-2DMCs permit the generation of S and I beams at higher operating temperatures in the weak coupling regime.[14] Still, as depicted in Fig. 1 d), S and I are degenerate in momentum space thus the preventing an easy spatial separation, while having $\mathrm{S}$ and I well separated in space is an important condition for a realistic source of twin beams. In addition, with this method, the PMCs impose a strictly equal energy spacing between the three photonic bands and the extraction efficiency of the I beam is poor, preventing the generation of separated and balanced beams.
In this paper we discuss a different opportunity for the generation of twin beams based on wire-shaped onedimensional microcavities (1D-MCs). The multiplicity of the 1D-MC photonic/polaritonic bands[15] and their fine structures[17] allow a versatile engineering of different scattering channels and of their polarization properties. The corresponding scheme is shown in Fig. 1 e). Interbranch parametric scattering can be obtained and the choice of horizontal processes (S and I degenerate in energy and split in momentum space) produces twin beams having intrinsically balanced intensities. The excitation is normal to the sample surface while S and I are mirrorsymmetric (see Fig. $1 \mathrm{f}$ )). The mode energy splitting is not a constraint (as it was in the case of triply-resonanat 2D-MCs) and the strong coupling requirement can be eventually relaxed.[13, 14, 16]

The paper is organized as follows: in Section 2 we describe the sample and the experimental methods; in Section 3 we show the results of polarization resolved measure- 
ments both under non-resonant and resonant excitation, at normal incidence and corresponding power dependence; finally the conclusions are drawn in Section 4.

2 Sample and experimental method The sample is realized in a molecular beam epitaxy reactor (MBE). It is formed by a $\lambda / 2$ 2D-MC $\left(\mathrm{Ga}_{0.05} \mathrm{Al}_{0.95} \mathrm{As}\right.$ [5]) sandwiched between two Bragg mirrors (respectively 26 pairs in the top and 30 pairs in the bottom made of $\left.\mathrm{Ga}_{0.05} \mathrm{Al}_{0.95} \mathrm{As} / \mathrm{Ga}_{0.80} \mathrm{Al}_{0.20} \mathrm{As}\right)$. Three stacks of four GaAs quantum wells (width $7 \mathrm{~nm}$ ) are placed at the antinodes of the cavity mode (one group at the cavity center and the other two at the first antinodes in each Bragg mirror). In order to finely tune the relative energy between the MC photonic mode at $\vec{k}=0\left(E_{2 D}\right)$ and the excitonic resonance $\left(E_{X}\right)$ a wedge in the layers thickness is created during the growth.

The 1D-MCs are then fabricated by reactive ion etching of the planar 2D-MC. Different set of 1D-MCs having width $L_{\perp}$ from $3 \mu \mathrm{m}$ to $7 \mu \mathrm{m}$ and same length $L_{\|}=1000$ $\mu \mathrm{m}$ are etched on the same sample. The investigation of a single nanoemitter is possible thanks to the relatively large spacing $(60 \mu \mathrm{m})$ between two adjacent 1D-MCs.

A tunable Ti:Sapphire laser provides a $\mathrm{CW}$ excitation; the beam is focused in a long and sharp spot $(\sim 100 \mu \mathrm{m}$ by $\sim 4 \mu \mathrm{m}$ in full width at half maximum). The laser polarization is set perpendicular to the wire axis (direction $\perp$ ). The sample is cooled to $10 \mathrm{~K}$ in a cold-finger, liquid-helium cryostat.

We investigate the far field radiation spectrum of single 1D-MCs by Fourier space imaging: the image of the back focal-plane of a micro-objective lens (NA $=0.4$, focal length $f=16 \mathrm{~mm}$ ) is focused on the exit slit of a 50 $\mathrm{cm}$ monochromator and detected by a silicon based CCD camera ( spectral resolution is $\sim 100 \mu \mathrm{eV}$ ).

In our experiment the 1D-MC long axis (direction $\|$ ) is set at $\sim 45$ degrees with respect to the entrance slit of the spectrometer making possible the simultaneous detection of the band dispersion of even and odd modes which in turn present an intensity maximum or a minimum at $\vec{k}=$ 0 . All the spectra are discriminated in polarization in order to detect the fine structure.

In 1D-MCs the refractive index has a discontinuity in the lateral direction perpendicular to the wire axis $(\perp)$ which influences the confinement of the electric field.[15] This discontinuity changes the photonic mode of the original 2D-MC in a set of modes indexed by $j=0,1,2, \ldots$ with energy at $\vec{k}=0$ given by $E_{1 D}^{j}=\sqrt{\left[E_{2 D}\right]^{2}+\left[\left(\hbar c / n_{C}\right)\left(\pi / L_{\perp}\right)(j+1)\right]^{2}}$ where $n_{C}$ is the refractive index of the cavity material.[18,19] For the sake of simplicity we refer to the detuning $\delta$ as the energy distance between $E_{X}$ and the energy of the lowest polariton mode $E_{P}^{j=0}, \delta=E_{X}-E_{P}^{j=0}$. All these modes have continuous dispersion along the direction parallel to
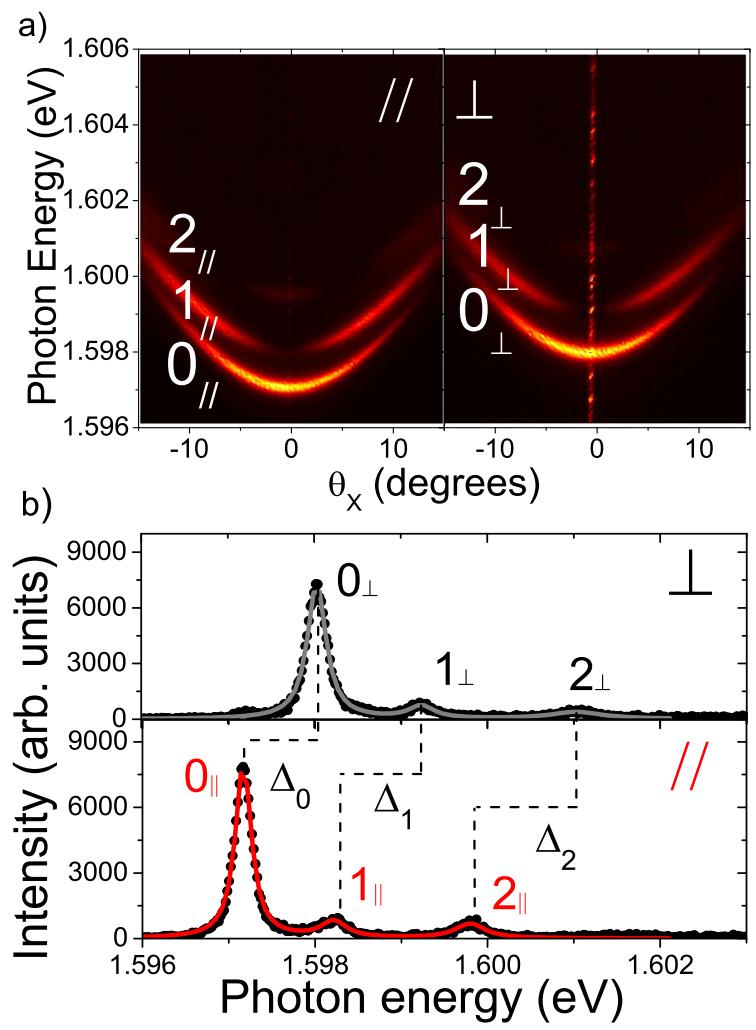

Figure 2 a) Color-intensity maps of a typical dispersion in energy-angle space of 4-nm 1D-MC at low temperature $(10 \mathrm{~K})$ and small detuning $(\delta \simeq-9 \mathrm{meV})$. Left (right) panel shows the polarization parallel (orthogonal) to the 1D-MC axis. b) 1D-MCs spectra as deduced by graph a) at $\theta_{Y} \simeq$ 5 degrees are shown in top and bottom panels for polarization orthogonal and parallel resepctively.

1D-MC $(\|)$ and are quantized in the orthogonal direction $(\perp)$.

3 Results and discussion Fig. 2 a) shows colourintensity maps of typical polariton modes emission $\left(L_{\perp}=4\right.$ $\mu \mathrm{m})$ under non resonant excitation and low power $\left(\mathrm{P}_{E x c} \simeq\right.$ $2 \mathrm{~mW}$ ) at $10 \mathrm{~K}$. The detected polarization in the left (right) panel is $\|(\perp)$ to the $1 \mathrm{D}-\mathrm{MC}$ axis.

Fig. 2 b) shows the two polarized spectra at $\theta_{Y} 5$ degrees (with this choice we can display the emission of modes with odd $j$ and eventually remove the unwanted laser stray light entering in the spectrometer). Polarization fine structures are present: each polariton mode is split in two sub-branches (with linear polarization \| and $\perp$ at lower and higher energy, respectively). The splitting $\left(\Delta_{j}=E_{P}^{j}(\perp)-E_{P}^{j}(\|)\right)$ was ascribed to mechanical stress due to the different thermal expansion coefficients of the copper cold finger of the cryostat (where the sample was glued at room temperature) and that of the GaAs 1D-MC sample.[17] 

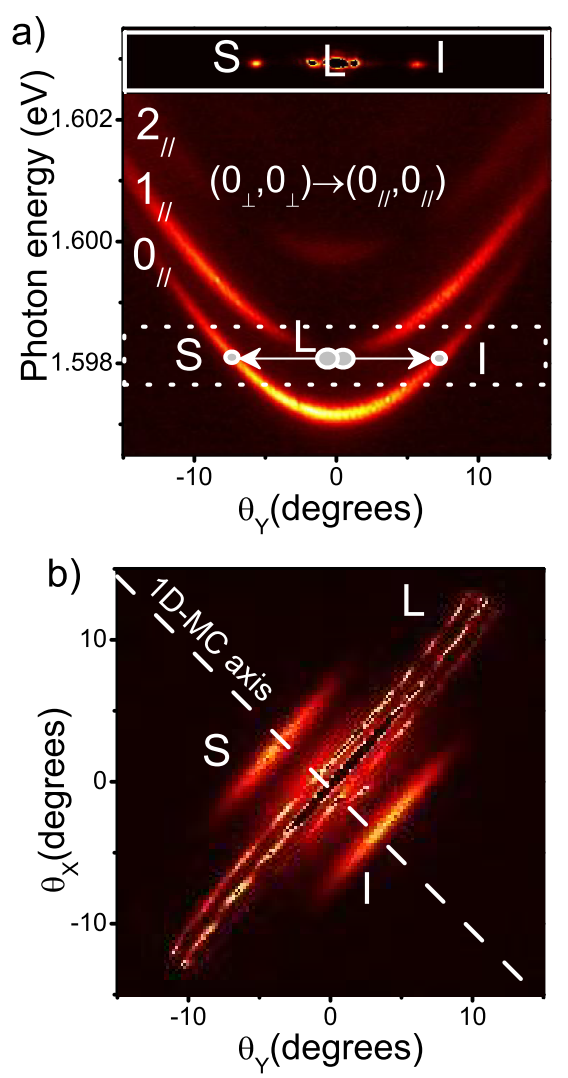

Figure 3 a) Energy-angle dispersion of a 1D-MCs representaed as a color intensity map. On top of the dispersion bands dots and arrows indicate the PSP $\left(\left(0_{\perp}, 0_{\perp}\right) \rightarrow\right.$ $\left.\left(0_{\|}, 0_{\|}\right)\right)$. The dashed area is represented in the inset at top under resonant excitation. b) S, L and I full far field in angle space represented as a color intensity map. The wire direction in realspace is also reported on the figure.

At $10 \mathrm{~K}$ the measured values of $\Delta_{j}$ increase with $j\left(\Delta_{0}\right.$ $=0.86 \mathrm{meV}, \Delta_{1}=1.02 \mathrm{meV}$ and $\Delta_{2}=1.23 \mathrm{meV}$ ). In previous studies the presence of the splittings $\Delta_{j}$ enabled both the study of polarization dynamics of polariton modes and the realization of vertical OPO, based on a linear polarization inversion mechanism.[17]

Let's now discuss how we can obtain twin beams in horizontal OPO by exploiting the same mechanism.

Fig. 3 a) shows a colour-intensity map of a 1D-MC dispersion for $\|$ polarization, obtained under non-resonant excitation. The arrows describe the scheme hereafter used for achieving PSPs. The value of $\Delta_{0}$ is larger with respect to the linewidth associated to the modes $0_{\perp}$ and $0_{\|}$(see Fig. 2 b)) and permits [17] the horizontal PSPs $\left(0_{\perp}, 0_{\perp}\right) \rightarrow\left(0_{\|}, 0_{\|}\right)$by resonantly exciting the $1 \mathrm{D}-\mathrm{MC}$ at the minimum of the $0_{\perp}$ band at $\vec{k}=0$ (see the arrows in Fig. 3 a)). The inset in Fig 3 a) shows the data obtained under resonant excitation : the pump (L), the signal (S) and the idler (I) produced trough the process just described

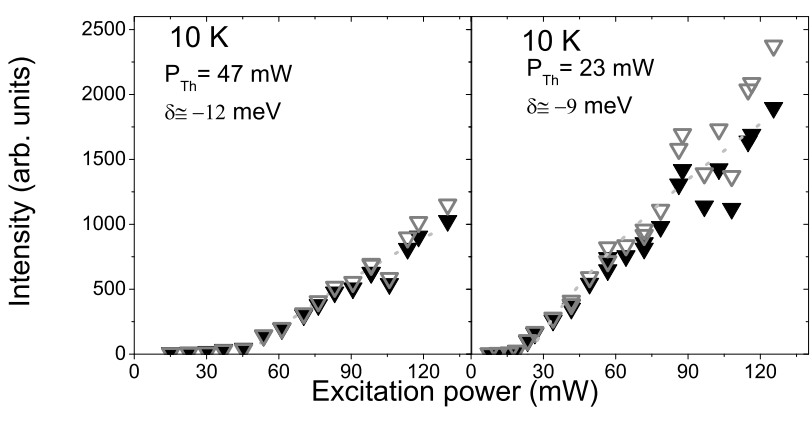

Figure 4 Left and right panels show S and I intensity as a function of the incident excitation power for two different 1D-MCs

are clearly resolved in angle. Fig 3 b) shows the far field emission from the sample. The two elongated and bright spots (S and I) are parallel to the laser spot (L) and perpendicular to the wire axis. The polarization of $\mathrm{S}$ and I are crossed with respect to the excitation laser accordingly to the fact that this scattering process is the only one allowed by PMCs.

A further indication of the parametric nature of the observed $\mathrm{S}$ and $\mathrm{I}$ in the $0_{\|}$mode can be obtained by studying the power dependence in the same resonant conditions. From the full far field images (see Fig. 3 b)) we measure the total intensity of $\mathrm{S}$ and $\mathrm{I}$ as a function of $\mathrm{P}_{E x c}$. The data extracted with this procedure are displayed in Fig. 4 for two different 1D-MCs. Both the processes present two distinct regimes: at low $\mathrm{P}_{\text {Exc }}$ the light emitted in the $0_{\|}$ mode is mainly composed by laser scattering and incoherent relaxation (barely linear with $\mathrm{P}_{E x c}$ ). Increasing $\mathrm{P}_{E x c}$ a threshold $\left(\mathrm{P}_{T h}\right)$ appears and above it the $\mathrm{S}$ and I emissions rapidly increase. By taking into account the dimension of the laser spot on the sample surface we estimate the measured $\mathrm{P}_{T h}$ in $\sim 12 \mathrm{KW} / \mathrm{cm}^{2}$ and $\sim 6 \mathrm{KW} / \mathrm{cm}^{2}$, for the left and right panel in Fig. 4 respectively. More generally the measured values of $\mathrm{P}_{T h}$ range between $\sim 1 \mathrm{KW} / \mathrm{cm}^{2}$ for $\delta \simeq-5 \mathrm{meV}$ up to $\sim 60 \mathrm{KW} / \mathrm{cm}^{2}$ for $\delta \simeq-20 \mathrm{meV}$. This finally demonstrates the generation of OPO with signal (S) and idler (I) beams with equal intensities, as well as relevant spatial separation.

4 Conclusions In conclusion we have shown the realization of OPO in 1D-MCs based on horizontal PSPs. The modes fan of 1D-MCs joined with large linear polarization splitting $\Delta_{j}$ permits an efficient engineering of the parametric scattering channels. The twin beams generated in horizontal processes are intrinsically balanced in intensity and well separated in momentum space. By polarization inversion mechanism we can set the polarization of $\mathrm{S}$ and I cross-polarized respect to the excitation laser. Note that the limit of $10 \mathrm{~K}$ for achieving OPO can be overcamed and higher operating temperatures could be in principle reached. In fact the present approach is not based on 
the strong coupling dispersion of lower polariton but on interbranch processes.

Acknowledgements V. A. and M.A. thank the european project EU Network ITN Clermont-4.

\section{References}

[1] A. V. Kavokin, J. J. Baumberg, G. Malpuech, and F. P. Laussy, Microcavities (Oxford University Press, New York, 2007)

[2] Y. R. Shen, The Principles of Nonlinear Optics (Wiley InterScience, Hoboken, 2003)

[3] J. A. Armstrong, N. Bloembergen, J. Ducuing, and P. S. Pershan, Phys. Rev. 127, 1918 (1962)

[4] K. Edamatsu, G. Oohata, R. Shimizu and T. Itoh Nat. (London) 431, 167 (2004).

[5] E. Wertz, L. Ferrier, D. D. Solnyshkov, P. Senellart, D. Bajoni, A. Miard, A Lemaitre, G. Malpuech, and J. Bloch, Appl. Phys. Lett. 95, 051108 (2009)

[6] E. Wertz, L. Ferrier, D. Solnyshkov, R. Johne, D. Sanvitto, A. Lemaitre, I. Sagnes, R. Grousson, A. V. Kavokin, P. Senellart, G. Malpuech and J. Bloch, Nature Physics, 6, 860 (2010).

[7] N.Gisin, G.Ribordy, W.Tittel, H.Zbinden, Rev. Mod. Phys.74, 145 (2002).

[8] D. Bouwmeester, A. K. Ekert, and A. Zeilinger, The Physics of Quantum Information: Quantum Cryptography, Quantum Teleportation, Quantum Computation (Springer-Verlag, Berlin, 2000)

[9] P. G. Savvidis, J. J. Baumberg, R. M. Stevenson, M. S. Skolnick, D. M. Whittaker, and J. S. Roberts, Phys. Rev. Lett. 84, 1547 (2000)

[10] M. Saba, C. Ciuti, J. Bloch, J. V. Thierry-Mieg, R. Andre, L. S. Dang, S. Kundermann, A. Mura, G. Bongiovanni, J. L. Staehli and B. Deveaud, Nat. 414, 731 (2003)

[11] J.J. Baumberg, P.G. Savvidis, R. M. Stevenson, A.I. Tartarovskii, M.S. Sckolnick, D.M. Whittaker, J.S. Roberts, Phys. Rev. B 62, 24 (2000)

[12] C. Diederichs and J. Tignon, Appl. Phys. Lett. 87, 251107 (2005)

[13] C. Diederichs, J. Tignon, G. Dasbach, C. Ciuti, A. Lemaitre, J. Bloch, Ph. Roussignol, and C. Delalande, Nature (London) 440, 904 (2006)

[14] D. Taj, T. Lecomte, C. Diederichs, Ph. Roussignol, C. Delalande, and J. Tignon Phys. Rev. B 80, 081308R (2009)

[15] G. Dasbach, M. Schwab, M. Bayer, D.N. Krizhanovskii, and A. Forchel, Phys. Rev. B 66, 201201R (2002)

[16] M. Abbarchi, V. Ardizzone, T. Lecomte, A. Lemaitre, I. Sagnes, P. Senellart, J. Bloch, Ph. Roussignol, and J. Tignon, Phys. Rev. B, 83, 201310 (2011)

[17] G. Dasbach, C. Diederichs, J. Tignon, C. Ciuti, Ph. Roussignol, C. Delalande, M. Bayer, and A. Forchel, Phys. Rev. B 71, 161308R (2005)

[18] C. Ciuti, Phys. Rev. B 69, 245304 (2004)

[19] A. I. Tartakovskii, V. D. Kulakovskii, A. Forchel, and J. P. Reithmaier, Phys. Rev. B 57, R6807 (1998) 